\title{
Molecular line emission from planetary and protoplanetary nebulae
}

\author{
Valentín Bujarrabal \\ Observatorio Astronómico Nacional, Apartado 112, E-28803 Alcalá de Henares, Spain \\ email: v.bujarrabal@oan.es
}

\begin{abstract}
Molecular line emission in the mm- and submm-wave domains is found to be a very useful tool to study planetary nebulae $(\mathrm{PNe})$. Molecular lines, particularly the low- $J$ transitions of carbon monoxide, are known to probe most of the nebular material in young PNe and protoplanetary nebulae (PPNe). Many quantitative results on these objects have been so obtained, including general structure, total mass and density distribution, kinetic temperatures, velocity fields, etc. In more evolved PNe, however, the molecular line intensity (or even detection) is quite uncertain, due to photodissociation. Molecular lines often trace just certain parts of these nebulae, like equatorial tori or axial clumps. Other molecules are in general more affected by photodissociation than $\mathrm{CO}$, but photo- or shock-induced chemistry produces high abundances of radicals and large molecules in some intermediate-evolution objects. The presence of rotating disks in some objects is finally discussed.
\end{abstract}

Keywords. stars: AGB and post-AGB; stars: mass loss; stars: winds, outflows; planetary nebulae: general; molecules; radio lines: stars.

\section{Introduction}

Molecular lines are very useful to study planetary nebulae (PNe), mainly, as we will see, young planetary nebulae or protoplanetary nebulae (PPNe). In particular, many important quantitative results are obtained from these observations.

The most important species to study PNe with is carbon monoxide; most of the results I will discuss in this review have been obtained from lines of this molecule. CO lines present important observational advantages: they are intense, due to the high abundance of this molecule, and can be observed with efficient radiotelescopes, yielding high spectral and spatial resolution.

The analysis of the observations of $\mathrm{CO}$ lines is moreover particularly reliable. We readily obtain information on the dynamics from such observations, precisely due to the high spectral resolution mentioned above. The chemistry of this molecule is very simple, with a high and quite constant abundance, except when photodissociation is important, then the molecule is rapidly destroyed (e.g. Mamon et al. 1988). So, CO lines are a good tracer of molecule-rich gas.

Finally, the excitation of the CO lines is particularly easy to describe. We are dealing with low-energy rotational lines within a single rotational ladder in the ground vibrational state (excited ones are usually too high in energy compared to the excitation state in molecular regions). For instance the most usually observed transitions are the $J=1-0$ and 2-1 lines, at mm-wavelengths, and the first rotational level $(J=1)$ is at just $5.5 \mathrm{~K}$ from the ground. These lines are moreover easily thermalized, due to their particularly low Einstein coefficients.

The total number of photons emitted by such low- $J$ transitions is approximately given, in the optically thin limit (and assuming that the mm-wave background temperature is 
much smaller than the line excitation temperature), by

$$
n_{p h}\left(\mathrm{~cm}^{-3} \mathrm{~s}^{-1}\right) \sim A n_{u} \sim A g_{u} n_{c o} e^{-E_{u} / k T_{\text {rot }}} / F_{\text {par }}\left(T_{\text {rot }}\right),
$$

where $\mathrm{A}$ is the Einstein coefficient of the transitions and $n_{u}, g_{u}$ and $E_{u}$ are the population, statistical weight and energy of its upper level. $F_{p a r}\left(T_{r o t}\right)$ is the partition function corresponding to a representative excitation temperature for the rotational ladder, which, as we have seen, takes values similar to the kinetic temperature, $T_{k}$. Note that the exponential factor is close to 1 , depending slowly on the excitation, since $E_{u}$ has low values in our case.

If we assume that these excitation temperatures are much larger than the level energies, this formula becomes :

$$
n_{p h} \stackrel{\propto}{\sim} n_{c o} / T_{k}=X_{c o} n_{t o t} / T_{k},
$$

where $X_{c o}$ is the relative CO abundance and $n_{t o t}$ is the total volume density.

Note that optical depth is often not negligible in CO lines, but it can be treated, using some escape probability formalism or, if possible, observing lines of the rarer isotope ${ }^{13} \mathrm{CO}$.

These formulae are just approximations, but they show that the dependence of the intensity on the physical conditions is smooth, just approximately proportional to the total mass and inversely proportional to the kinetic temperature. In fact, these approximations are known to be not very inaccurate, and some of the results we will see below are obtained from simplified descriptions of this kind.

\section{Molecular line surveys in PNe}

Pioneering surveys of CO emission in PNe were performed by Huggins and coworkers (1996). More recently, these works have been continued, particularly including protoplanetary nebulae (e.g. Bujarrabal et al. 2001).

Huggins et al. showed that the mass ratio between molecule-rich and ionized material depends a lot on the nebular radius, most of the material being molecular in compact sources. However, a strong dispersion appeared in the data. This is partially due to the ill-definition and difficult measurement of the size of the nebula. It is also clear that other stellar parameters should be taken into account; in particular, the total nebular mass in objects with low initial mass seems to be very low.

In Fig. 1, I have represented the total molecular mass of PNe and PPNe as a function of their age, which is taken to be equal to their post-AGB kinematic lifetimes (including data from Huggins et al. and more recent results). As we can see, the dispersion is still very high. We can discuss some particular cases to see its origin. For instance, M 2-9 is a low-luminosity source, probably with low initial mass. Its ionized and neutral atomic masses are not high (Castro-Carrizo et al. 2001), so certainly the total nebular mass in this object remains low, $\sim 0.05 \mathrm{M}_{\odot}$. The dashed line represents what would happen if we assume that the distance usually taken for this source $(\sim 650 \mathrm{pc})$ is wrong and we increase it to bring its luminosity up to a 'normal' value. In any case, the strong dependence of the total mass on the initial mass can also be seen in other objects; I have noted in the figure the case of R Sct, an RV Tau variable, i.e. probably a low-mass post-AGB source.

The uncertainty in the nebular age is also large, since kinematic times are often difficult to interpret. Note the case of NGC 7662, for example. The triangle shows a reliable measurement of the kinematic time (from proper motions by Hajian and Terzian 1996), but the dashed line represents here other ages that could be deduced from values of the distance, size and velocity found in the bibliography (e.g. Pottasch 1984). 


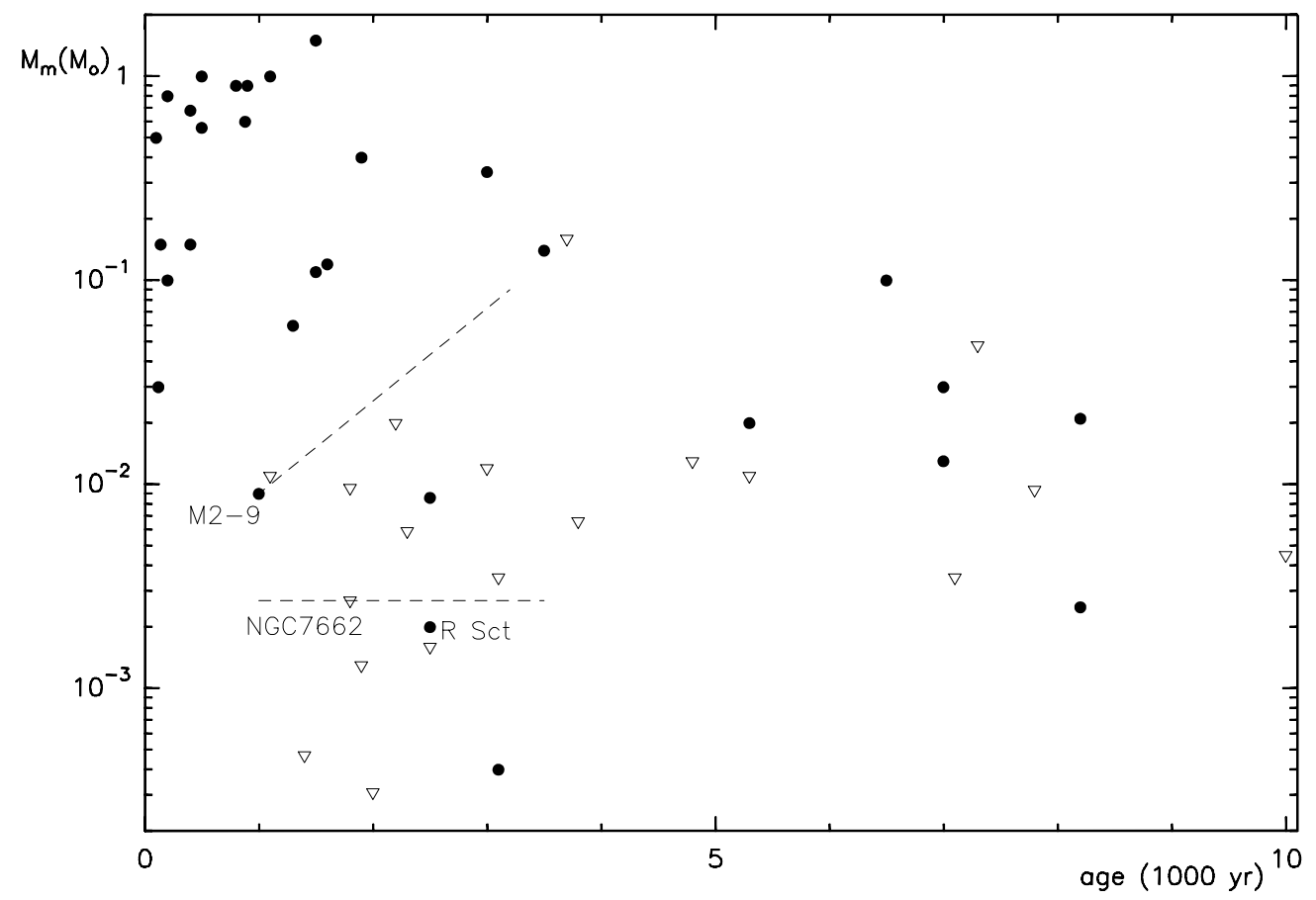

Figure 1. Molecular mass measured from CO line observations of PNe versus nebula age, i.e. post-AGB kinematic times. Empty symbols represent mass upper limits. Molecular data come from: Huggins et al. (1996, 2000, 2004), Bujarrabal et al. (2001), Castro-Carrizo et al. (2001, 2002), Fong et al. (2001), Sánchez Contreras et al. (2004, 2006), Forveille et al. (1998), Zweigle et al. (1997). Nebular lifetimes come from Pottasch (1984), Hajian and Terzian (1996) and the above papers.

In spite of all these uncertainties, two results appear clearly from Fig. 1. First, for relatively evolved nebulae, the total amount of molecular gas is strongly variable from object to object, and often relatively low, with a lot of nondetections. However, for young $\mathrm{PNe}$ (or PPNe), younger than $\lesssim 1000 \mathrm{yr}$, there is a significant population of nebulae in which molecular lines probe masses larger than $0.1 \mathrm{M}_{\odot}$. In some objects, the mass of the molecule-rich component is close to $1 \mathrm{M}_{\odot}$. This means that CO lines probe most of the nebular gas in young $\mathrm{PNe}$, indeed, in many of them this molecular component seems to represent most of the initial mass of the source, now ejected by the central star.

\section{Relatively evolved planetary nebulae}

We have seen that the intensity of molecular lines from relatively evolved PNe, even their detection, is quite uncertain. But not only the intensity of the lines varies a lot from object to object. Also their spatial distribution, the part of the nebula that the molecular gas represents, is difficult to predict. Very probably, important photodissociation effects are present (Sect. 6).

An example of recent results on this topic is the observations of $\mathrm{CO} J=2-1$ in $\mathrm{BD}+30$ 3639 by Bachiller et al. (2000). CO lines come mainly from two compact clumps that seem to be placed along the axis of the torus-like structure defined by the ionized gas emission. Both clumps are escaping along that axis at high velocity $\left(\gtrsim 60 \mathrm{~km} \mathrm{~s}^{-1}\right)$. The total detected mass is low $\left(\lesssim 0.01 \mathrm{M}_{\odot}\right)$; we note that apparently there is also some CO 
emission at the central velocities lost in these data, it probably corresponds to some residual molecular gas associated to the ionized torus.

An important case is that of the Helix Nebula, NGC 7293, see Young et al. (1999), Huggins et al. (2002). The overall distribution of the CO line emission is very similar to the well known optical image, although CO only probes about $1 / 10$ of the total mass. The velocity information obtained allowed in particular a detailed description of the nebula shape in three dimensions (a couple of rings seen almost face-on).

The detection of $\mathrm{CO}$ emission from the cometary globules in the Helix is very interesting. $\mathrm{CO}$ seems to come from the whole globule, with a total mass of about $10^{-5} \mathrm{M}_{\odot}$. CO lines show a particularly low velocity dispersion across each globule, less than $1 \mathrm{~km} \mathrm{~s}^{-1}$ and comparable to the expected thermal velocity dispersion; this result confirms that cometary globules are not associated to shock activity. I note that a talk by P. Huggins in this session deals in detail with such interesting structures.

A different kind of molecular line source is $\mathrm{KjPn} 8$. This source shows a wide, elongated structure in the optical image, occupying more than 10 arcmin. Almost perpendicularly to its main axis, there is a second one, probably associated to a more recent ejection. $\mathrm{CO}$ emission is detected in a tiny structure $\left(\sim 20^{\prime \prime}\right.$ wide $)$ that seems to correspond to a torus almost exactly perpendicular to the secondary, new jet (Forveille et al. 1998). The total mass of this molecular ring is however not negligible, $0.03 \mathrm{M}_{\odot}$. We will see that other examples of multiple axes seen in the optical have also a counterpart in molecular emission.

Other interesting recent studies of molecular lines from evolved PNe are the detection of the equatorial dense disk in M1-16 (Huggins et al. 2000) and the complementary survey of PNe by Huggins et al. (2005).

\section{Young planetary nebulae}

The situation is very different when we deal with protoplanetary nebulae (PPNe). The CO line emission often probes the whole nebula and detects large amounts of mass, between $0.3-1 \mathrm{M}_{\odot}$. The maps show nebulae with a clear axial symmetry. In most PPNe, bipolar outflows are found along that axis, together with equatorial disk-like structures. The velocities of the bipolar flows are high, up to $400 \mathrm{~km} \mathrm{~s}^{-1}$, often showing a clear velocity gradient with the distance to the star ('Hubble-like velocity law') that suggests ballistic expansion; a significant post-AGB acceleration has probably taken place in that direction. However, the disks are almost always in moderate expansion, at $\sim 10 \mathrm{~km} \mathrm{~s}^{-1}$; in these structures the post-AGB dynamics has had little effect. (As we will see, there are a few disks with high expansion velocities and one or two disks in rotation.)

In some sources, the kinetic temperature can be estimated from line ratios. In most of them, the excitation is low $(10-30 \mathrm{~K})$, but we will also see sources with significantly higher temperatures.

A relatively old but enlightening result is the CO mapping of M 1-92 (Bujarrabal et al. 1998 and references there in). The CO nebula occupies the whole optical image. Two empty shells flow mostly along the symmetry axis, with a velocity that increases linearly with distance up to $\sim 70 \mathrm{~km} \mathrm{~s}^{-1}$. There is also a central ring in slow expansion. The shape and velocity field of the double shell strongly suggest that it has been accelerated by bow shocks propagating along the axial direction.

Detailed modeling of the source shows that both components are quite dense (between $5 \times 10^{4}$ and $\left.3 \times 10^{5} \mathrm{~cm}^{-3}\right)$ and cold $(10-15 \mathrm{~K})$. Such density and velocity distributions yield very high linear momenta along the axis, $310^{39} \mathrm{~g} \mathrm{~cm} \mathrm{~s}^{-1}$, much larger than what radiation pressure can release under expected conditions. 

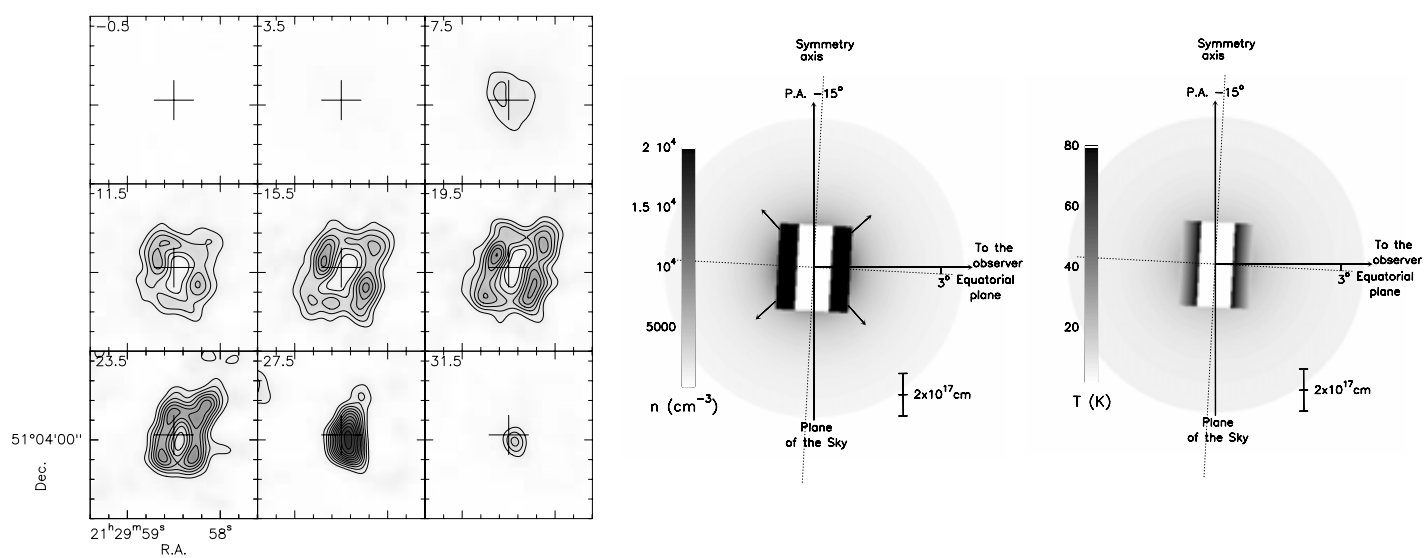

Figure 2. CO $J=2-1$ observations of IRAS $21282+5050$ (maps per velocity channel, LSR velocity is indicated in the upper-left corners) and model that reproduces these data. Note the high temperature gradient across the walls of the dense cylinder. (From the new PdBI survey of AGB and post-AGB sources, Castro-Carrizo et al., in preparation.)

Particularly interesting are also the cases of $\mathrm{OH} 231.8+4.2$ and Frosty Leo, both presenting high mass and momentum and low temperature. $\mathrm{OH} 231.8+4.2$ shows a bipolar flow with velocities up to $400 \mathrm{~km} \mathrm{~s}^{-1}$ and a remarkably constant velocity gradient; the flow is very elongated in the direction of the axis of the complex optical nebula (Alcolea et al. 2001). Frosty Leo is an example of multiple axes. This nebula shows an axis of symmetry at large scale but, at small scale, jets in other directions appear; one of them is particularly conspicuous and seems formed by a series of bow shocks. The CO emission (Castro-Carrizo et al. 2005) is associated to this second axis, with a fast outflow in its direction. A torus-like structure showing a high velocity dispersion appears in the perpendicular plane.

Probably the most detailed model developed for a PPN is that of CRL 618, by SánchezContreras et al. (2004); the various components of the model correspond to features actually detected in their accurate mapping. There are two slow components: a halo corresponding to a mass-loss process more or less similar to those typical of obscured AGB stars, plus a very compact and dense one, corresponding to a mass-loss rate as strong as $2 \times 10^{-4} \mathrm{M}_{\odot} \mathrm{yr}^{-1}$, but lasting only about $500 \mathrm{yr}$. Two fast axial outflows are found: a double shell, apparently formed by axial shocks, plus a very fast inner outflow, running inside the cavity left by the shells. The temperature of these components is anomalously high, several hundred K, probably due to recent heating by shocks.

Finally, I mention the unpublished observations of IRAS $21282+5050$, belonging to the new Plateau de Bure Interferometer (PdBI) survey of AGB and post-AGB sources (Fig. 2; Castro-Carrizo et al., in preparation). Most of the gas is concentrated in a cylinderlike structure in moderate expansion. We note the high temperature gradient across the walls of the the cylinder, necessary to explain the high contrast between the intensity at positive and negative relative velocities. In this case the heating is probably due to UV photons, since there is a well developed PDR in the center (Fong et al. 2001).

Among other interesting observations of PPNe, I note first the maps of objects sharing the above mentioned properties (a central component plus bipolar fast outflows that carry relatively high mass and momentum): HD 101584 (Olofsson \& Nyman, 1999), M 256 (Castro-Carrizo et al., 2002), Hen 3-1475 (Huggins et al., 2004), IRAS19475 + 3119 (Sánchez Contreras et al., 2006), IRAS 22036+5306 (Sahai et al., 2006, in preparation), 
etc. However, CRL 2688 was found to show a peculiar structure of jets at various orientations (Cox et al. 2000). Finally, I note that only a few detailed submm-wave observations (higher- $J$ CO lines) have been performed, namely, the maps of CRL 618 in CO $J=6-5$ (Nakashima et al., 2006, in preparation), showing emission from the central components of the nebula, and the $J=4-3$ low-resolution data of several objects by Hrivnak \& Bieging (2005). Hrivnak \& Bieging (2005) found slightly smaller masses than other authors. However, they analyzed their data assuming nebula properties similar to those of AGB circumstellar envelopes, including spherical symmetry; their models are then quite different from those discussed above and difficult to compare with results from other papers.

\section{Dynamics of PPNe: Statistics}

The accurate maps mentioned in the previous section, together with systematic singledish observations, have allowed statistical studies of the properties of PPNe from CO data; see e.g. Bujarrabal et al. (2001).

If we consider all PPNe (i.e. young PNe with stellar temperatures not higher than 30000 $\mathrm{K}$ ) that have been relatively well studied in CO emission, we end up with a total of 30 or 40 nebulae. More than 2/3 of these show a high total molecular gas mass, between about 0.1 and $1 \mathrm{M}_{\odot}$. In them, we can conclude that CO probes most of the nebular material, sometimes most of the initial mass. In the sources in which we can estimate the ejection time from the extent and velocity of the $\mathrm{CO}$ emitting gas, i.e. the time during which the mass was ejected by the star (presumably in the late AGB phases), we usually find short values of a few thousand years: the ejection of most of the material of PNe seems then to be a very fast phenomenon.

Of well studied PPNe, only $\sim 15 \%$ do not show fast outflows down to reasonable detection limits. Of course, a good deal of these cases could be due to projection effects (jet directions close to the sky plane), high photodissociation in extended structures, or peculiar nebulae (for instance, sources with low initial mass or probably containing rotating disks are among them). Typically, about $0.1 \mathrm{M}_{\odot}$ are found to have been strongly accelerated during a (probably) very short post-AGB phase.

In more than $80 \%$ of the sources with useful CO data, the momentum carried by the high-velocity flows is too high to be driven by radiation pressure, at least under expected conditions (even taking into account the effects of multiple scattering in opaque shells). Probably, another mechanism should be invoked; see some discussion in Sect. 7.

\section{Chemical evolution in $\mathrm{PNe}$}

I have mentioned that the chemistry of PNe is dominated by the evolution from the very molecule-rich AGB circumstellar envelopes to the ionized evolved PNe. Such a process is thought to be mostly due to photodissociation and ionization by the UV photons of the increasingly hot central star, which penetrate a nebula that is becoming more and more diffuse. Shock-induced chemistry and dissociation by interstellar photons are also important in some nebulae. During this process, other molecules less abundant than CO are in general more easily photodissociated, due to self-shielding effects. PDRs rich in neutral atoms can also be present and even be the dominant component of the nebulae. See works by Huggins et al. (1996, 2002, 2005), Bujarrabal et al. (1994), Zweigle et al. (1997), Young et al. (1999), Bachiller et al. (1997a,b, 2000), Castro-Carrizo et al. (2001, 2002), Fong et al. (2001), Woods et al. (2005), Cernicharo et al. (2004), etc.

In some intermediate-evolution nebulae, we detect radicals (notably $\mathrm{CN}$ and $\mathrm{HCO}^{+}$) and more rarely large molecules (carbon chains, benzene, etc). The presence of these 
species is due to the dissociation of stable molecules and the very active chemistry characteristic of radical-rich gas, before these radicals and new molecules are themselves dissociated.

An example of O-rich nebulae presenting such a rich chemistry is $\mathrm{OH} 231.8+4.2$, which shows surprisingly high abundances of $\mathrm{HCO}^{+}, \mathrm{HNC}, \mathrm{SiO}$, etc. In this nebula, showing very high axial velocities, shock-induced chemistry could be dominant (SánchezContreras et al. 1997, 2000).

The most remarkable case is that of the carbon-rich PPN CRL 618. CRL 618 shows an incredibly rich spectrum in the $\mathrm{mm}$ and submm domains, including lines of molecules like $\mathrm{HC}_{5} \mathrm{~N}, \mathrm{HC}_{7} \mathrm{~N}, \mathrm{H}_{2} \mathrm{O}$, benzene, etc. Both lines from the ground and excited vibrational states are found. See e.g. Cernicharo et al. (2001), Cernicharo (2004), Pardo et al. (2005) and references therein; there is also a very interesting poster in this symposium on this source (Pardo et al.). Models explain the presence of all these molecules assuming photoinduced chemistry. It is remarkable that these models are very compatible with those explaining the CO maps (Sect. 4), though often the outer parts of the nebula are not detected in rare molecules.

\section{Rotating disks around post-AGB stars}

Many PPNe show disk-like structures in the equatorial plane of the nebula. In all cases we have seen up to now, these disks are found to be in expansion. However, the existence of rotating disks have been invoked to explain the collimated and energetic jets from post-AGB stars, in a similar way as they are expected to be involved in the formation of jets in very young stars. See theoretical developments in Soker (2002), Frank \& Blackman (2004), García-Segura et al. (2005), as well as in the contributions by A. Frank and G. García-Segura in this symposium. Rotating disks could also explain certain properties sometimes found in post-AGB objects, like the detection of hot dust grains (which should require a stable reservoir of grains close to the star), the relative lack of refractory elements in the stellar atmospheres (due possibly to reaccretion of nebular material), and the peculiar profiles found in a few PPNe (difficult to explain for standard expanding nebulae). See as general reference Van Winckel (2003).

Only one of such rotating disks has been clearly detected in PPNe. CO $J=2-1$ and $J=1-0$ maps of the Red Rectangle (Bujarrabal et al. 2003, 2005) show a structure elongated along the equatorial plane of the nebula, in which a thick dust disk was known to be present. The $\mathrm{CO}$ velocity-position diagrams in the equatorial direction show clearly the signature of keplerian rotation. Models of $\mathrm{CO}$ emission confirm that the disk is in rotation, and that the rotation is keplerian at least in the inner $\sim 810^{15} \mathrm{~cm}$. The rotation dynamics corresponds to a central mass (probably the mass of the binary central star) of $\sim 1.5 \mathrm{M}_{\odot}$.

Outside that radius, the disk is still rotating, but a very slow expansion (at less than $1 \mathrm{~km} \mathrm{~s}^{-1}$ ) also appears. This low expansion velocity, comparable to the thermal velocity dispersion, suggests that the expansion is just due to density gradients or to some kind of evaporation. This is supported by the fact that the models tend to predict a significant decrease of the density at the radius at which the dynamics change, by a about a factor three.

I finally recall that it is still not clear if such structures are prevalent in the inner regions of PPNe and could explain the ubiquitous post-AGB axial jets, since only one of them has been well detected up to date. 


\section{Acknowledgements}

This work has been supported by the Spanish Ministerio de Ciencia y Tecnología, under grant number ESP2003-04957.

\section{References}

Alcolea, J., Bujarrabal, V., Sánchez Contreras, C., Neri, R., \& Zweigle, J. 2001, A\&3A 373, 932

Bachiller, R., Forveille, T., Huggins, P.J., \& Cox, P. 1997a, A\&̈A 324, 1123

Bachiller, R., Fuente, A., Bujarrabal, V., et al. 1997b, A\&A 319, 253

Bachiller, R., Forveille, T., Huggins, P.J., Cox, P., \& Maillard, J. P. 2000, A $\& A$ 353, L5

Bujarrabal, V., Fuente, A., \& Omont, A. 1994, A\&A 285, 247

Bujarrabal, V., Alcolea, J., \& Neri, R. 1998, ApJ 504, 915

Bujarrabal, V., Castro-Carrizo, A., Alcolea, J., Sánchez \& Contreras, C. 2001, A\&A 377, 868

Bujarrabal, V., Neri, R., Alcolea, J., \& Kahane, C. 2003, A $\xi A$ 409, 573

Bujarrabal, V., Castro-Carrizo, A, Alcolea, J., \& Neri, R. 2005, A\&A 441, 1031

Castro-Carrizo, A., Bujarrabal, V., Fong, D., et al. 2001, A $\& A$ 367, 674

Castro-Carrizo, A., Bujarrabal, V., Sánchez Contreras, C., Alcolea, J., \& Neri, R. 2002, A\&A 386, 633

Castro-Carrizo, A., Bujarrabal, V., Sánchez Contreras, C., Sahai, R., \& Alcolea, J. 2005, A\&A 386, 633

Cernicharo, J., Heras, A.M., Tielens, A.G.G.M., et al. 2001, ApJ 546, L123

Cernicharo, J. 2004, ApJ 608, L41

Cox, P., Lucas, R., Huggins, P.J., et al. 2000, A $\mathscr{E} A$ 353, L25

Fong, D., Meixner, M., Castro-Carrizo, A., et al. 2001, ApJ 367, 652

Forveille, T., Huggins, P.J., Bachiller, R., \& Cox, P. 1998, ApJ 495, L111

Frank, A. \& Blackman, E.G. 2004, ApJ 614, 737

García-Segura, G., López, J. A., \& Franco, J. 2005, ApJ 618, 919

Hajian, A.R. \& Terzian, Y. 1996, PASP 108, 258

Hrivnak, B.J. \& Bieging J.H. 2005, ApJ 624, 331

Huggins, P.J., Bachiller, R., Cox, P., \& Forveille, T. 1996, A $\& A$ 315, 284

Huggins, P.J., Forveille, T., Bachiller, R., \& Cox, P. 2000, ApJ 544, 889

Huggins, P.J., Forveille, T., Bachiller, R., Cox, P., Ageorges, N., \& Walsh, J.R. 2002, ApJ 573, L55

Huggins, P.J., Muthu, C., Bachiller, R., Forveille, T., \& Cox, P. 2004, A\&A 414, 581

Huggins, P.J., Bachiller, R., Planesas, P., Forveille, T., \& Cox, P. 2005, ApJS 160, 272

Mamon, G.A., Glassgold, A.E., \& Huggins, P.J. 1988, ApJ 328, 797

Olofsson, H. \& Nyman, L-Å 1999, A\& A 347, 1940

Pardo, J.R., Cernicharo, J., \& Goicoechea, J.R. 2005, ApJ 628, 275

Pottasch, S.R. 1984, Planetary Nebulae - A study of late stages of stellar evolution D. Reidel, Dordrecht

Sánchez Contreras, C., Bujarrabal, V., \& Alcolea, J. 1997, A\&A 327, 689

Sánchez Contreras, C., Bujarrabal, V., Neri, R., \& Alcolea, J. 2000, A\&A 357, 651

Sánchez Contreras, C., Bujarrabal, V., Castro-Carrizo, A., Alcolea, J., \& Sargent, A. 2004, ApJ 617, 1142

Sánchez Contreras, C., Bujarrabal, V., Castro-Carrizo, A., Alcolea, J., \& Sargent, A. 2006, ApJ, in press

Soker, N. 2002, ApJ 568, 726

Young, K., Cox, P., Huggins, P.J., Forveille, T., \& Bachiller, R. 1999, ApJ 522, 387

Van Winckel, H. 2003, ARA\& A 41, 391

Woods, P.M., Nyman, L., Schöier, F.L., Zijlstra, A.A., Millar, T.J., \& Olofsson, H. 2005, A\&A 429,977

Zweigle, J., Neri, R., Bachiller, R., Bujarrabal, V., \& Grewing, M. 1997, A $\&$ A 324, 624 

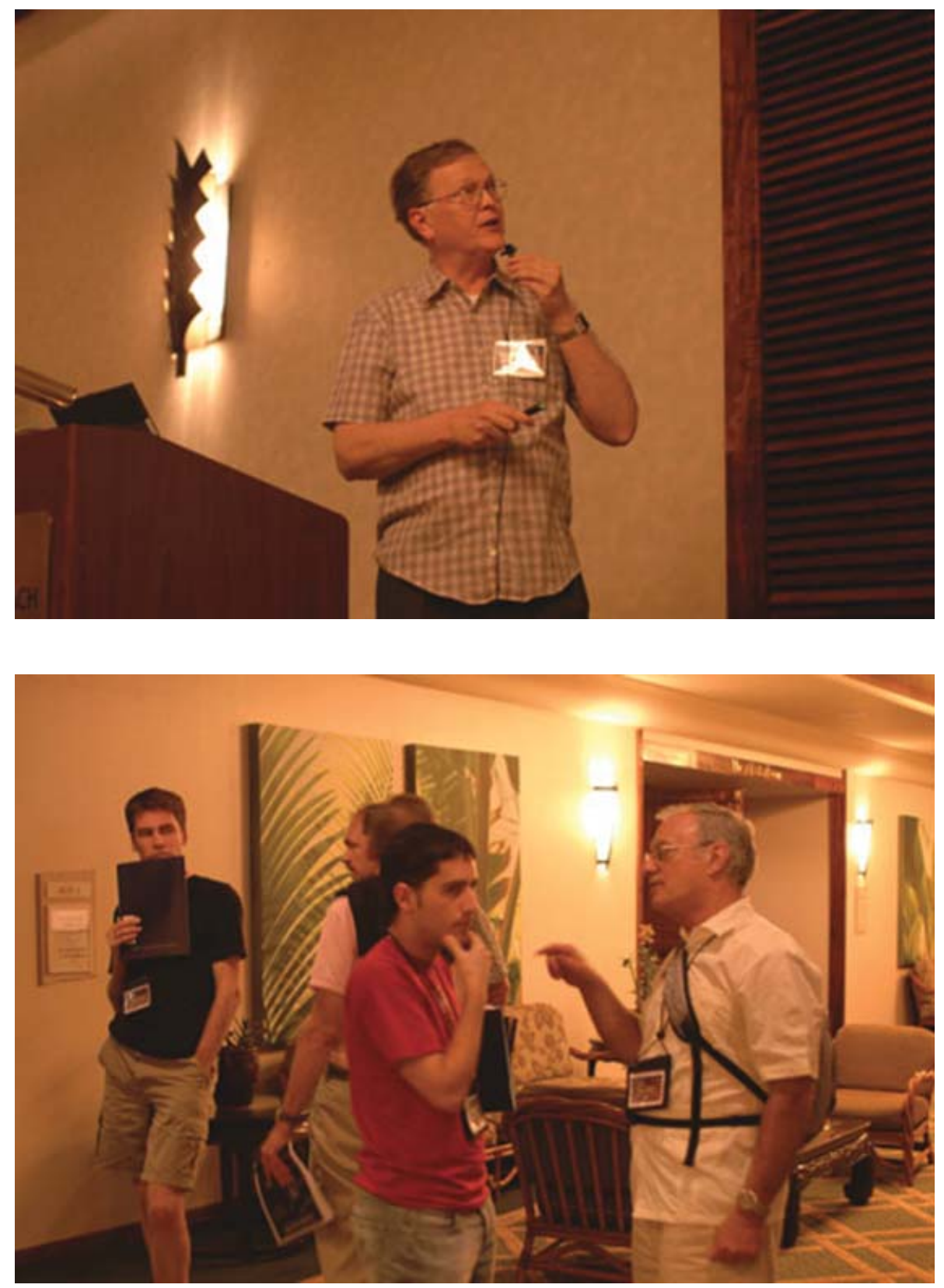

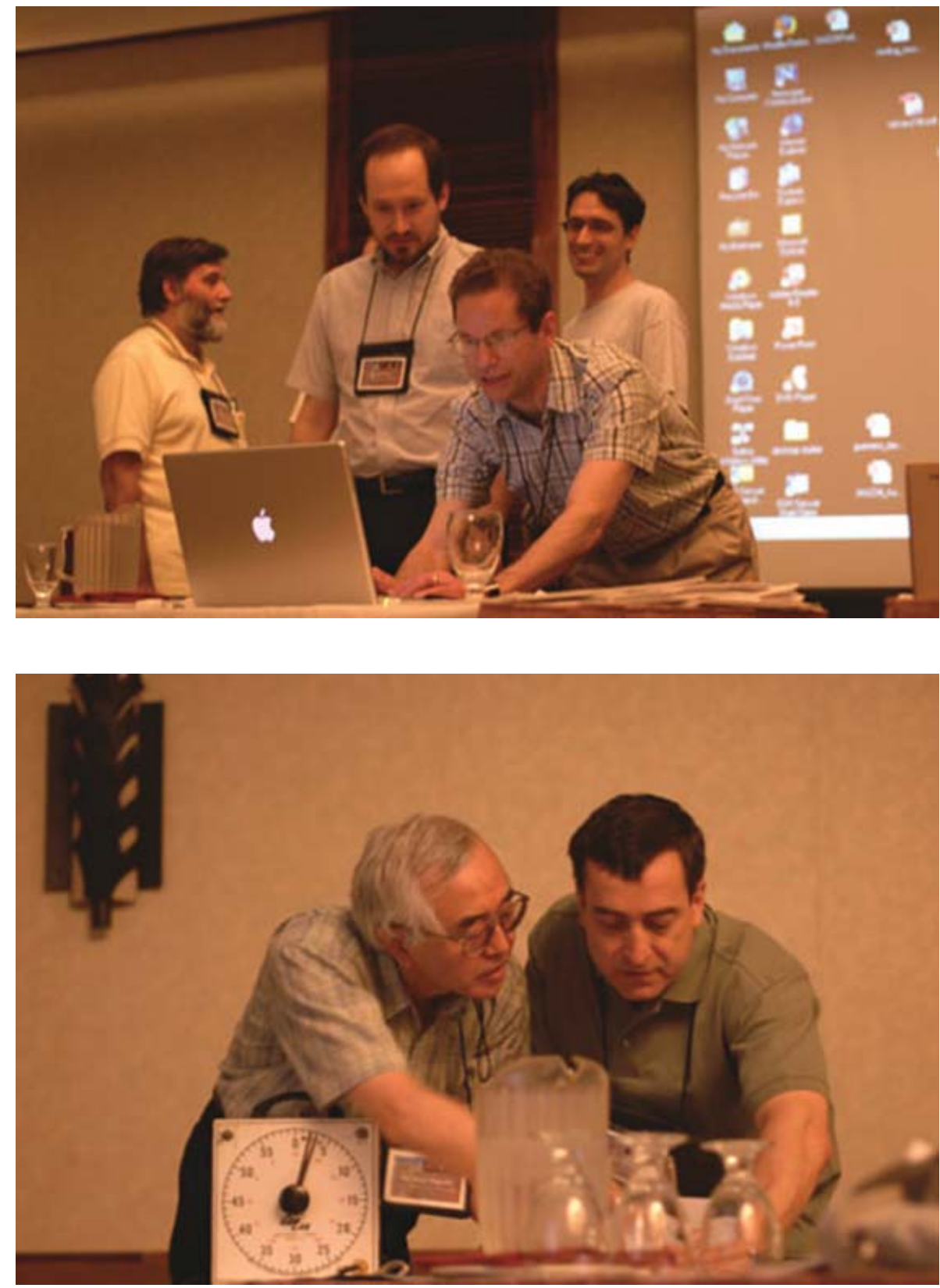